\title{
Liver transplantation of a patient with extreme thrombocytopenia - A case report -
}

Received January 5, 2021

Revised May 8, 2021

Accepted May 17, 2021

\author{
Yena Oh' ${ }^{1}$, Seung Yeon Yoo', Gyu-Seong Choi ${ }^{2}$, and Gaabsoo Kim ${ }^{1}$ \\ Departments of ${ }^{1}$ Anesthesiology and Pain Medicine, ${ }^{2}$ Transplantation Surgery, Samsung \\ Medical Center, Sungkyunkwan University School of Medicine, Seoul, Korea
}

Background: Patients with chronic liver disease (CLD) planned for liver transplantation (LT) often show severe thrombocytopenia, but there is a lack of evidence in deciding the threshold for prophylactic platelet transfusion.

Case: A 47-year-old female with acute liver failure was referred for LT. Despite daily transfusion of platelets, platelet counts remained under 10,000/ $\mu$ l. During LT, 2 units of single donor platelets (SDP) were transfused. Although platelet counts remained extremely low $(3,000-4,000 / \mu \mathrm{l})$ no diffuse oozing was observed and the blood loss was $860 \mathrm{ml}$. Postoperatively, there was no sign of active bleeding or oozing, and the patient received only 1 unit SDP transfusion.

Conclusions: CLD patients may have severe thrombocytopenia. However, primary hemostasis may not be significantly hindered due to the existence of rebalanced hemostasis. Prophylactic platelet transfusion in these patients should not be decided based on platelet counts only, but also take other coagulation tests and clinical signs into consideration.

Keywords: Liver transplantation; Platelet transfusion; Rebalanced hemostasis; Thrombocytopenia.
Thrombocytopenia is frequently observed in patients with chronic liver disease (CLD), with a prevalence of up to $76 \%$ [1].

Although mild in most cases, severe thrombocytopenia (platelet count below 50,000/ $\mu$ l) is not uncommon. Traditionally, severe thrombocytopenia has been considered a definite indication for prophylactic platelet transfusion before major surgery such as liver transplantation (LT) [2]. However, since the introduction of rebalanced hemostasis, thrombocytopenic patients with CLD have been thought to be more resistant to low platelet counts in terms of bleeding risk [3]. Patients undergoing LT often show severe thrombocytopenia, but there is lack of evidence to support the threshold for prophylactic platelet transfusion. We report the case of a patient with very severe thrombocytopenia who underwent LT with minimal platelet transfusion. This case report was approved by the Institutional Review Board (no. 2020-03-061-003) and written informed consent was obtained from the patient for the publication of this case.

\section{CASE REPORT}

A 47-year-old female (body weight $49.6 \mathrm{~kg}$, height $159 \mathrm{~cm}$ ) suffered from acute liver failure due to alcoholic hepatitis. The patient was a heavy drinker and was hospitalized due to mental changes two months prior to LT. She received steroid treatment for severe alcoholic hepatitis, but it was tapered

This is an Open Access article distributed under the terms of the Creative Commons Attribution Non-Commercial License (http://creativecommons.org/licenses/by-nc/4.0) which permits unrestricted non-commercial use, distribution, and reproduction in any medium, provided the original work is properly cited.

Copyright (C) the Korean Society of Anesthesiologists, 2021 
due to lack of response. Treatment for hepatic encephalopathy and endoscopic variceal obturation for gastric variceal bleeding was performed as well. One week before LT, she was intubated due to dyspnea caused by pulmonary edema, probably due to excessive transfusion and fluid treatment after hematemesis.

She was transferred to our hospital six days prior to LT. Initial laboratory results were hemoglobin $8.7 \mathrm{~g} / \mathrm{dl}$, hematocrit $26 \%$, platelet count 2,000/ $\mu \mathrm{l}$, prothrombin time (PT) $29.0 \mathrm{~s}$ with an international normalized ratio (INR) 2.73, and activated partial thromboplastin time (aPTT) 49.9 s. Computed tomography revealed liver cirrhosis, splenomegaly, and multiple collateral vessels in the abdominopelvic cavity. Esophagogastroduodenoscopy revealed large gastric varices and portal hypertensive gastropathy. Echocardiography was normal. The patient had very severe thrombocytopenia; despite daily transfusion of 6 to 18 units of leukocyte-depleted platelet concentrate (LDPC), platelet counts remained under $10,000 / \mu \mathrm{l}$. However, there was no spontaneous bleeding despite persistent severe thrombocytopenia. In the intensive care unit (ICU), along with transfusion, ventilator care and continuous renal replacement therapy was done until LT.

A deceased donor liver was allocated and the intubated patient was transferred to the operating room. After standard monitoring, general anesthesia was induced with intravenous thiopental sodium $50 \mathrm{mg}$, atracurium $50 \mathrm{mg}$, and sevoflurane. The right radial artery, right femoral artery, and right femoral vein were cannulated for monitoring and sampling. A 9-Fr catheter (ARROW MAC, Teleflex, USA) was inserted into the right internal jugular vein and a pulmonary artery catheter was inserted to monitor pulmonary artery pressure, continuous cardiac output, and mixed venous oxygen saturation. Anesthesia was maintained with oxygen, air, isoflurane, and atracurium.

On the day of surgery, only 2 units of single donor platelets (SDPs) were available in the hospital. Therefore, we planned to transfuse the 2 units of SDPs throughout the surgery. Perioperative coagulation profiles and non-activated thromboelastometry (NATEM) results are shown in Table 1. Despite continuous transfusion of platelets, platelet count remained at 3,000-4,000/ $\mathrm{ll}$. Although platelet count was very low, no diffuse oozing was observed in the operating field and blood loss expressed as lost red cell mass was $860 \mathrm{ml}$ [4]. Crystalloid 3,050 ml, 5\% albumin $550 \mathrm{ml}, 4$ units of packed red blood cells, $550 \mathrm{ml}$ of autotransfusion, 7 units of fresh frozen plasma, and 2 units of SDPs were infused during the 300-min LT procedure. Continuous infusion of norepinephrine $(0.01-0.2 \mu \mathrm{g} / \mathrm{kg} / \mathrm{min})$ was maintained throughout the surgery and the recipient was transferred to the ICU.

Postoperatively, there was no active bleeding or oozing, and the recipient received only 1 unit of SDPs on the day after surgery. Although thrombocytopenia due to peripheral sequestration and destruction by splenomegaly was suspected, laboratory tests and consultation with the department of hemato-oncology were performed to determine the cause of persistent very severe thrombocytopenia. Many spherocytes and schisticytes (2-3/high-power field) were observed in peripheral blood smear. Positive direct antiglobulin test and increased D-dimer suggested immune hemolytic thrombocytopenia. Bone marrow biopsy was recommended to confirm whether thrombocytopenia was peripheral in origin rather than from production failure. Also, con-

Table 1. Coagulation Profiles and Non-activated Thromboelastometry (NATEM) Results during and after Liver Transplantation

\begin{tabular}{|c|c|c|c|c|c|c|c|c|c|}
\hline Time of sampling & $\begin{array}{l}\text { Platelets } \\
(1,000 / \mu \mathrm{l})\end{array}$ & PT (INR) & aPTT (s) & $\begin{array}{c}\text { Fibrinogen } \\
\text { (mg/dl) }\end{array}$ & $\mathrm{CT}(\mathrm{s})$ & $\mathrm{CFT}(\mathrm{s})$ & $\begin{array}{c}\alpha \text { angle } \\
\text { (degree) }\end{array}$ & $\mathrm{MCF}(\mathrm{mm})$ & LI 60 (\%) \\
\hline Preoperation & 3 & 2.19 & 56.8 & 110 & - & - & - & - & - \\
\hline After induction & 3 & 2.36 & 58.9 & 55 & 2,729 & NA & 7 & 5 & 100 \\
\hline Anhepatic start & 4 & 2.17 & 62.3 & 82 & 1,402 & NA & 11 & 15 & 90 \\
\hline 5 min after reperfusion & 3 & 2.33 & 113.3 & 84 & $1,246 *$ & $N A^{*}$ & $9 *$ & $14 *$ & $70 *$ \\
\hline 1 hour after reperfusion & 4 & 2.23 & 106.9 & 97 & Flat & Flat & Flat & Flat & Flat \\
\hline End of operation & 8 & 2.05 & 69.4 & 117 & - & - & - & - & - \\
\hline POD 1 & 4 & 1.19 & -15.2 & 292 & 1,601 & 6,561 & - & 21 & 100 \\
\hline POD 5 & 16 & 1.05 & -13.7 & 170 & 1,029 & 1,071 & 15 & 28 & 100 \\
\hline POD 6 & 31 & - & - & - & 996 & 736 & 21 & 30 & 98 \\
\hline
\end{tabular}

PT (INR): prothrombin time (international normalized ratio) (reference range: 0.80-1.30), aPTT: activated partial thromboplastin time (reference range: 30-40 s), CT: clotting time (reference range: 300-1,000 s), CFT: clot formation time (reference range: 150-700 s), a angle: alpha angle (reference range: $30-70^{\circ}$ ), MCF: maximum clot firmness (reference range: 40-65 mm), LI 60: lysis index 60 (reference range: 76-96\%), POD: postoperative day. NA: not available. *Protamine-treated Fig. 1. Perioperative changes in platelet count. 
sultation recommended that if thrombocytopenia persists, splenectomy may be required. However, without further treatment, platelet counts started to increase from postoperative day (POD) 4 and spontaneously recovered to over $100,000 / \mu \mathrm{l}$ on POD 10 (Fig. 1). On POD 20, the patient was discharged without major complications.

\section{DISCUSSION}

To the best of our knowledge, this case reports the lowest preoperative platelet counts for a patient undergoing LT. There have been reports of patients with very severe thrombocytopenia undergoing extrahepatic mass excision (19,000/ ul) [5] or laparoscopic splenectomy ( $1,000 / \mathrm{ul})$ [6], but LT is usually considered to be more complex with a greater risk of bleeding, and thus a more challenging procedure.

In CLD patients, thrombocytopenia is a very common complication with multifactorial origins. An increase in splenic sequestration due to hypersplenism, a decrease in thrombopoietin production, an increase in platelet destruction due to autoantibodies, and an increase in platelet consumption due to cirrhosis-related hypercoagulability all can play a role $[7,8]$. Although thrombocytopenia in these patients may be severe, this is to some extent compensated for by an increase in von Willebrand factor (vWF) and a decrease in a disintegrin and metalloprotease with thrombospondin type 1 motif 13 (ADAMTS13) resulting in rebalanced hemostasis, thus reducing the risk of bleeding $[7,9,10]$.

During LT, 2 units of SDPs were transfused slowly starting just after the induction of anesthesia until the end of surgery. Although platelet counts were under 10,000/ $\mu \mathrm{l}$ throughout surgery, no diffuse oozing was observed in the operating field. Platelet counts remained under $10,000 / \mu$ for 5 days after LT, and there was no evidence of significant postoperative bleeding. This may reflect that in CLD patients with very severe thrombocytopenia, primary hemostasis may not be as impaired as expected. There seems to be little evidence to suggest a threshold for platelet transfusion in these patients [10], and therefore further studies are warranted.

Platelet count reaches its nadir at POD 6, and then starts to increase [11]. This is in line with what we observed in the present case. Platelet count remained steady at $4,000 / \mu \mathrm{l}$ after LT until it started to rise dramatically, reaching $126,000 / \mu \mathrm{l}$ at POD 10. As portal hypertension is resolved after LT, platelet count increases and hypersplenism is reversed [12]. Thromboelastomerty results support this. Maximum clot firmness [MCF], which reflects platelet count, improved along with all other indicators at POD 6. Even when platelet counts were very low, lysis index 60 (LI 60) was relatively normal, which might contribute to lowering bleeding tendency by maintaining clot stability. The fibrinolytic system may also be in balance in patients with CLD, due to the concomitant decrease in antifibrinolytics (antiplasmin, thrombin activatable fibrinolysis inhibitor), and plasminogen [10]. Thus, in

Platelet counts, blood

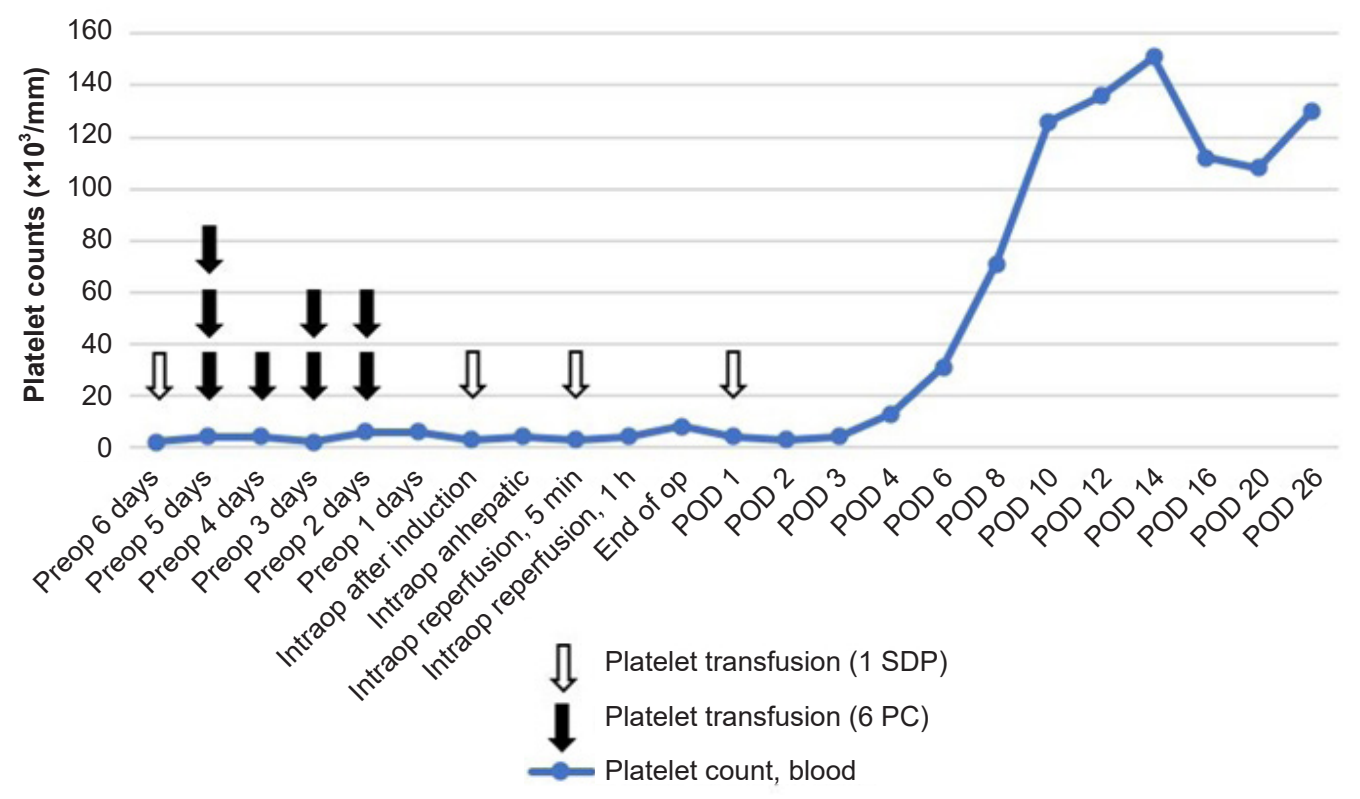

Fig. 1. Perioperative platelet counts. SDP: single donor platelets, PC: platelet counts, POD: postoperative day. 
CLD patients, conventional coagulation tests such as PT, aPTT, and platelet count may not fully reflect complex changes in the coagulation system. Although it is difficult to confirm the presence of rebalanced hemostasis, thromboelastography (TEG) or rotational thromboelastometry (ROTEM) may be helpful in assessing coagulopathy in these patients [5]. Also, clinical signs such as bruising, purpura, epistaxis, gingival bleeding, menorrhagia, and bleeding associated with invasive procedures may exist in the absence of rebalanced hemostasis.

Although platelet transfusion is the mainstay of clinical management of severe thrombocytopenia, the platelet count at which transfusions are indicated remains controversial in CLD patients undergoing invasive procedures. Generally, according to transfusion guidelines, platelets are given routinely before interventions in uncomplicated thrombocytopenic patients with a count of less than $10,000-20,000 / \mu \mathrm{l}$ $[2,13]$. Since patient platelet count in the present case were consistently under $10,000 / \mu \mathrm{l}, 6$ to 18 units of LDPC were transfused daily before LT, but platelet count remained under $10,000 / \mu \mathrm{l}$. This correlates with findings that the platelet counts of CLD patients with thrombocytopenia do not typically increase significantly after platelet transfusion [14]. We also transfused 2 units of SDPs during LT and this, along with rebalanced hemostasis, may have had a role in hemostasis during surgery. Nevertheless, immune hemolytic thrombocytopenia was not clearly ruled out and prophylactic platelet transfusions may not provide additional benefit in this patient, it would have been worthwhile trying less platelet transfusion in perioperative period. It was difficult to determine the cause of thrombocytopenia in this case. However, considering that the platelet count did not rise even after multiple blood transfusions, and normalized without any specific treatment after LT, it can be assumed that hypersplenism due to portal hypertension was the main cause of thrombocytopenia rather than production failure or other immunologic reactions.

Although the practice of transfusion has become very safe, there still are some risks that clinicians should be aware of, especially in LT patients, including infection, alloimmunization, and febrile and nonhemolytic reactions. Apart from the possibility of developing inflammatory reactions or bacterial/viral infections, platelet transfusion during LT is suspected to increase postoperative mortality due to acute lung injury [15]. Therefore, the potential benefit and risk of harm should be judged by clinicians making decisions regarding platelet transfusion.
In conclusion, even in CLD patients with very severe thrombocytopenia, primary hemostasis may not be significantly hindered. Prophylactic platelet transfusion in these patients should not be decided based only on platelet count, but should also take into account other coagulation tests and clinical signs of bleeding.

\section{CONFLICTS OF INTEREST}

No potential conflict of interest relevant to this article was reported.

\section{DATA AVAILABILITY STATEMENT}

Data sharing not applicable to this article as no datasets were generated or analyzed during the current study.

\section{AUTHOR CONTRIBUTIONS}

Conceptualization: Yena Oh, Seung Yeon Yoo, Gyu-Seong Choi, Gaabsoo Kim. Methodology: Yena Oh, Seung Yeon Yoo, Gaabsoo Kim. Writing - original draft: Yena Oh. Writing - review \& editing: Yena Oh, Seung Yeon Yoo, Gyu-Seong Choi. Supervision: Seung Yeon Yoo, Gyu-Seong Choi, Gaabsoo Kim.

\section{ORCID}

Yena Oh, https://orcid.org/0000-0002-2764-9075

Seung Yeon Yoo, https://orcid.org/0000-0002-7226-4238

Gyu-Seong Choi, https://orcid.org/0000-0003-2545-3105

Gaabsoo Kim, https://orcid.org/0000-0002-9383-2652

\section{REFERENCES}

1. Giannini EG. Review article: thrombocytopenia in chronic liver disease and pharmacologic treatment options. Aliment Pharmacol Ther 2006; 23: 1055-65.

2. Estcourt LJ, Birchall J, Allard S, Bassey SJ, Hersey P, Kerr JP, et al. British Committee for Standards in Haematology. Guidelines for the use of platelet transfusions. Br J Haematol 2017; 176: 365-94.

3. Saab S, Brown RS Jr. Management of thrombocytopenia in patients with chronic liver disease. Dig Dis Sci 2019; 64: 2757-68.

4. Bang SR, Ahn HJ, Kim GS, Yang M, Gwak MS, Ko JS, et al. Predictors of high intraoperative blood loss derived by simple and objective method in adult living donor liver transplantation. Transplant Proc 2010; 42: 4148-50. 
5. Han B, Lee SY, Kim GS. Anesthetic management of a chronic liver disease patient with very low platelet counts by considering a rebalanced hemostasis - a case report -. Anesth Pain Med 2018; 13: 419-22.

6. Wu Z, Zhou J, Pankaj P, Peng B. Laparoscopic splenectomy for immune thrombocytopenia (ITP) patients with platelet counts lower than $1 \times 10^{9} / \mathrm{L}$. Int J Hematol 2011; 94: 533-8.

7. Aster RH. Pooling of platelets in the spleen: role in the pathogenesis of "hypersplenic" thrombocytopenia. J Clin Invest 1966; 45: 645-57.

8. Afdhal N, McHutchison J, Brown R, Jacobson I, Manns M, Poordad F, et al. Thrombocytopenia associated with chronic liver disease. J Hepatol 2008; 48: 1000-7.

9. Feys HB, Canciani MT, Peyvandi F, Deckmyn H, Vanhoorelbeke K, Mannucci PM. ADAMTS13 activity to antigen ratio in physiological and pathological conditions associated with an increased risk of thrombosis. Br J Haematol 2007; 138: 534-40.

10. Potze W, Porte RJ, Lisman T. Management of coagulation abnormalities in liver disease. Expert Rev Gastroenterol Hepatol
2015; 9: 103-14.

11. Chang FY, Singh N, Gayowski T, Wagener MM, Mietzner SM, Stout JE, et al. Thrombocytopenia in liver transplant recipients: predictors, impact on fungal infections, and role of endogenous thrombopoietin. Transplantation 2000; 69: 70-5.

12. Witte M, Langnas AN, Hirst K, Stratta RJ, Shaw BW Jr. Impact of liver transplantation on the reversal of hypersplenism. Transplant Proc 1993; 25: 1987.

13. Peck-Radosavljevic M. Thrombocytopenia in chronic liver disease. Liver Int 2017; 37: 778-93.

14. Hirooka M, Ochi H, Hiraoka A, Koizumi Y, Tanaka T, Sunago K, et al. Role of severe thrombocytopenia in preventing platelet count recovery in thrombocytopenic patients with chronic liver disease. J Gastroenterol Hepatol 2020; 35: 299-304.

15. Pereboom IT, de Boer MT, Haagsma EB, Hendriks HG, Lisman T, Porte RJ. Platelet transfusion during liver transplantation is associated with increased postoperative mortality due to acute lung injury. Anesth Analg 2009; 108: 1083-91. 